\title{
Bone Marrow Extracted Autologous Mononuclear Cell Therapy in Autism
}

\author{
Dr. Vijay Rajesh Andanamala ${ }^{1}$, Dr. Naga Sankar Vemuri ${ }^{2}$ \\ ${ }^{1}$ M Tech-Bio-Technology, Ph.D., House No: 1-2-89/4A; SVN Colony- 7/2, Guntur-522006, AP, India \\ ${ }^{2}$ M.S., M.Ch. (Neurosurgery), Raghavendra Hospitals, Opp. Street to Sivalayam, D.No. 12-14-2, Kothapet, Guntur-522001, AP, India
}

\begin{abstract}
Autism is a disorder which is considered as complex development disorder. It caused due to neurological trauma that has direct effect on normal brain function. Symptoms are characterized by very less social interaction, improved verbal or nonverbal communications and indulgence in repetitive behaviour. We present a child with autism. She underwent autologous bone marrow extracted mononuclear cell therapy with a mean of $12 \times 10^{6}$ mono nuclear cells. Post therapy the child had no advance events and her clinical course after the therapy was uneventful. In the time period of 2 months we noticed reduced sensory integration problems, motor skills were improved to some extent, social skills were improved normalization in reflexes, stoppage of disease progression.
\end{abstract}

Keywords: Autism, Autologous, Mononuclear cells

\section{Introduction}

Autism spectrum disorder (ASD) is a complex developmental disability; signs typically appear during early childhood and affect a person's ability to communicate, and interact with others. ASD is defined by a certain set of behaviors and is a "spectrum condition" that affects individuals differently and to varying degrees. There is no known single cause of autism, but increased awareness and early diagnosis/intervention and access to appropriate services/supports lead to significantly improved outcomes. Some of the behaviors associated with autism include delayed learning of language; difficulty making eye contact or holding a conversation; difficulty with executive functioning, which relates to reasoning and planning; narrow, intense interests; poor motor skills' and sensory sensitivities. Again, a person on the spectrum might follow many of these behaviors or just a few, or many others besides. The diagnosis of autism spectrum disorder is applied based on analysis of all behaviors and their severity.

\section{Case Report}

At 8 years' female child who was born of consanguineous marriage had a Caesarian -Section delivery at maternity center. After the delivery immediately the child's weight is normal. At age of 6 years' she was diagnosed with as autism child.

She showed impaired verbal communications and indulgence in repetitive behavior. Her reflexes were not normal. She had attention deficit hyperactivity disorder. MRI was done but it showed no abnormality and EEG was also done which revealed no abnormality. Thus on the basis of clinical presentation she was diagnosed as autism child. At present the is no treatment for autism. So autologous mononuclear cell therapy was the only option in regenerative medicine.

So her parents were explained about this autologous mononuclear cell transplantation and they accepted it. Informed consent was taken from the parents which permitted us to do autologous mono nuclear cell transplantation.Pre-operative blood investigations such as Haemogram with pathologist opinion, Random Blood Sugar, Renal Function Tests, Liver Function Tests, Complete Viral Markers, Bleeding Time \& Clotting Time were conducted and the results were within the normal biological limits and Complete Urine Analysis was done which revealed no pathology, ultra sound abdomen was taken by the radiologist which revealed no structural and functional abnormality.

Bone marrow $(100 \mathrm{ml})$ was aspirated from both iliac bones by pathologist under intra venous sudation (Propofol + Ketamine with oxygen supplementation by anesthetist using a standard procedure. Then the autologous mononuclear cells were separated and isolated under strict aseptic condition using density gradient centrifugation by doctorate in Bio-Technology and approximately $12 \times 10^{6}$ autologous mononuclear cells were injected intrathecally into L3,L4 space using an epidural set and catheter by Neurosurgeon. Granulocyte colony stimulating factor injections were given subcutaneously to this child 24 hours prior therapy and 48 hours after therapy and methylprednisolone administered during the transplantation helped in stimulation of CD34 + cells and also in their survival and multiplication and antibiotic was given 10 days to the child following the therapy. Following the therapy she took speech therapy, occupational therapy as a part of treatment

\section{Discussion}

Autism spectrum disorder was caused by different combination of genetic and environmental influences. Autism most obvious signs tend to appear between $2 \& 3$ Years of age. In some cases it can be diagnoses as early as 18 months some development delays associated with autism can be identified and addressed even earlier.

There is no known cure for autism. Treatment proposed are to ease the symptoms and keep the patient functional as long as possible. Our case show autism futures like very low social interaction, no normal reflexes, Indulgence in repetitive behaviour. Post autologous stem cell therapy had

\section{Volume 6 Issue 12, December 2017}




\section{International Journal of Science and Research (IJSR) \\ ISSN (Online): 2319-7064}

Index Copernicus Value (2016): 79.57 | Impact Factor (2015): 6.391

side effects and her clinical course after therapy is uneventful. We have followed the case after therapy for 21 months. Normality in reflexes were observed, there was improvement in motor skills to some extent within 2 months of after therapy. There was stoppage in progression of disease.

We have demonstrated the possibility of use of autologous hematopoietic mononuclear cells (Stem Cells) in autism. Further clinical trials are required to demonstrate the effect of autologous hematopoietic mononuclear cells (Stem Cells) as a supportive assistance for autism

\section{References}

[1] Autism / Pervasive developmental disorder assessment and intervention for young children (Publication No 4217)

[2] International journal of language and communication disorder 47 (3) 233-244

[3] American psychiatric association (2013) diagnostic and statistical manual of mental disorders ( $5^{\text {th }}$ Edition) Washington DC

[4] Gray .C White AL \& Mc Andrew S (2002) By social stories book Philadelphia, PA: Jessica Kingsley Publishers 